\title{
Virial coefficients and vapor-liquid equilibria of the EXP6 and 2-Yukawa fluids
}

\author{
J. Krejčị, I. Nezbedd $2^{112}$, R. Melnyk ${ }^{3}$, A. Trokhymchuk ${ }^{3}$ \\ ${ }^{1}$ Faculty of Science, J. E. Purkinje University, 40096 Ústí nad Labem, Czech Republic \\ 2 Institute of Chemical Process Fundamentals, Academy of Sciences, 16502 Prague 6, Czech Republic \\ 3 Institute for Condensed Matter Physics of the National Academy of Sciences of Ukraine, \\ 1 Svientsitskii Str., 79011 Lviv, Ukraine
}

Received 26 April 2011, in final form 11 May 2011

\begin{abstract}
Virial coefficients $B_{2}$ through $B_{4}$ and the vapor-liquid equilibria for the EXP6 and 2-Yukawa (2Y) fluids have been determined using numerical integrations and Gibbs ensemble simulations, respectively. The chosen $2 Y$ models have been recently determined as an appropriate reference fluid for the considered EXP6 models.
\end{abstract}

Key words: EXP6 fluid, 2-Yukawa fluid, virial coefficients, vapor-liquid equilibrium, critical point

PACS: $05.20,51.30$

\section{Introduction}

Simple fluids, i.e., the fluids whose molecules interact via a spherically symmetric potential, $u=u(r)$, are most commonly modeled as Lennard-Jones (LJ) fluids. When applied to simple real fluids, the LJ performs reasonably well at subcritical and slightly supercritical conditions. However, for quite obvious reasons it fails at high temperatures/pressures: Repulsive interactions at these conditions are very soft and it has been well established that the EXP6 potential provides much more faithful description of the intermolecular interactions [1]. Furthermore, the exponential repulsion agrees with molecular beam scattering data as is also known from theoretical studies. It is therefore highly desirable, particularly from the point of geochemical and industrial (conditions of detonations and propagation of shock waves) applications, to reach a similar level of understanding and theoretical description of the EXP6 fluids similar to that of the LJ fluid.

A number of simulation data for the EXP6 fluid are available in literature along with early theoretical attempts (see [2] and references therein). The problem of theory is that all common methods are based on the assumption of the presence of very steep repulsions at short separations and thus make use of, either directly or indirectly, the known properties of the fluid of hard spheres. Consequently, they are not applicable to models with soft repulsion. To overcome this problem we have recently developed an alternative to HS-based theories, a theory based on the Yukawa (Y) model as a reference [3]. The Y potential seems to be a 'universal' simple fluid model because it is possible, by changing its parameter [see equation (2) below], to change both its range and repulsive softness. This is the reason why the Y potential is used in applications to describe of a variety of physical phenomena (see [4] and references therein).

The Y potential is a model belonging to the family of van der Waals models, i.e., the model with a hard core and approximating interactions outside the core. A large body of results, both theoretical and simulation, is available in literature (see, e.g., references [5-7] and references therein) for the Y fluid. However, to apply the Y potential to more realistic models with a soft repulsive part (i.e., without a hard core), it is necessary to use a combination of two (or even more) Y functions which results in a model without any hard core.

In a recent paper [2] we investigated, by means of molecular simulations, the structure of the EXP6 fluids and formulated the criteria for determining a Y model [more accurately, two Yukawa 
model $(2 \mathrm{Y})$ ] which could be used as a reference system for developing an analytic theory of the EXP6 fluids. Unlike the one Y model (1Y), multiple Y models have not been investigated in detail yet and only a handful of results are available [8, 9]. To accomplish the goal, i.e., to develop an analytic theory for the EXP6 fluid, we should first know the properties of the $2 \mathrm{Y}$ fluids accurately and in detail and this has been the motivation for the present study.

Since the virial coefficients provide the basic information on the properties of the fluid and can be used in various theoretical methods, we computed the first four virial coefficients of both the parent EXP6 fluid and descending 2Y fluids. Furthermore, we have also determined the vapor-liquid equilibrium (VLE) of the $2 \mathrm{Y}$ fluid and located the critical point which is an important information for setting the criteria of determining the $2 \mathrm{Y}$ fluid associated with the EXP6 fluid.

\section{Basic definitions and computational details}

The EXP6 fluid is a collection of additive species (atoms, molecules, etc.) interacting via the EXP6 potential (also referred to as a modified Buckingham potential),

$$
u_{\mathrm{EXP} 6}(r)= \begin{cases}\infty, & \text { for } r<r_{\max }, \\ \epsilon\left(\frac{6}{\alpha-6} \exp \left[\alpha\left(1-r / r_{\mathrm{m}}\right)\right]-\frac{\alpha}{\alpha-6}\left(r_{\mathrm{m}} / r\right)^{6}\right), & \text { for } r>r_{\max }\end{cases}
$$

where $r_{\max }$ and $r_{\mathrm{m}}$ is the location of the potential maximum and minimum, respectively, and $\epsilon$ is the depth of the minimum. Parameters $r_{\mathrm{m}}$ and $\epsilon$ are used henceforth as the length and energy units; dimensionless number density, $\rho^{*}$, temperature, $T^{*}$, pressure, $P^{*}$, and internal energy, $U^{*}$, are thus defined as $\rho^{*}=\rho r_{\mathrm{m}}^{3}, T^{*}=T k_{\mathrm{B}} / \epsilon, P^{*}=P r_{\mathrm{m}}^{3} / \epsilon$, and $U^{*}=U / \epsilon$, respectively, where $k_{\mathrm{B}}$ is the Boltzmann constant.

The hard core $1 Y$ potential is defined as

$$
u_{\mathrm{Y}}(r)= \begin{cases}+\infty, & \text { for } r<\sigma \\ (\sigma / r) \exp (-z r), & \text { for } r \geqslant \sigma\end{cases}
$$

where $z$ is the parameter governing the range of the interaction.

The $2 \mathrm{Y}$ potential is a model made up of two Yukawa tails without, in general, any hard core,

$$
u_{2 Y}(r)=\epsilon_{1} \frac{\sigma}{r} \exp \left[-\kappa_{1} r\right]-\epsilon_{2} \frac{\sigma}{r} \exp \left[-\kappa_{2} r\right]
$$

where $\epsilon_{1}>0$ and $\epsilon_{2}>0$ are the strengths of the repulsive and attractive contributions, respectively, while $\kappa_{1}^{-1}$ and $\kappa_{2}^{-1}$ are the measures of the range of the corresponding tails.

The virial coefficients, $B_{i}$, of the virial expansion,

$$
\frac{P}{\rho k_{\mathrm{B}} T}=1+\sum_{i>1} B_{i} \rho^{i-1}
$$

were evaluated numerically up to $B_{4}$ over a wide range of temperatures using the Mayer sampling [10]. We have recently examined another version of the virial expansion, the perturbed expansion around a suitable reference system similarly to the theories of liquids,

$$
\frac{P}{\rho k_{\mathrm{B}} T}=\left(\frac{P}{\rho k_{\mathrm{B}} T}\right)_{\mathrm{ref}}+\sum_{i>1}\left[B_{i}(T)-B_{i, \mathrm{ref}}(T)\right] \rho^{i-1}
$$

where subscript "ref" refers to a reference system. To determine the VLE envelope we used the common Gibbs ensemble with the total number of particles $N=512$ and applied the long-range correction in order to truncate the potential at $r_{\mathrm{c}}=0.45 \sqrt[3]{N / \rho^{*}}$. 


\section{Results and discussion}

When the EXP6 model is applied to real fluids by adjusting its parameter $\alpha$, its resulting values typically vary between 11 and 15. To keep contact with our previous papers [2, 11] we have chosen the bracketing values, $\alpha=11.5$ and 14.5. Parameters of the $2 \mathrm{Y}$ model descending from these EXP6 models were determined in our previous paper [2] and are given in table 1 .

Table 1. The parameters of the $2 \mathrm{Y}$ potential function defined by equation (3) which are used to represent the EXP6 fluid.

\begin{tabular}{llrrrrr}
\hline & $\alpha$ & $\epsilon_{1} / \epsilon$ & $\kappa_{1} r_{m}$ & $\epsilon_{2} / \epsilon$ & $\kappa_{2} r_{m}$ & $\sigma / r_{\mathrm{m}}$ \\
\hline SET I & 11.5 & 15026.86 & 9.4548 & 227.61 & 4.650 & 0.872 \\
SET II & 14.5 & 389565.31 & 13.344 & 148.24 & 4.514 & 0.892 \\
\hline
\end{tabular}
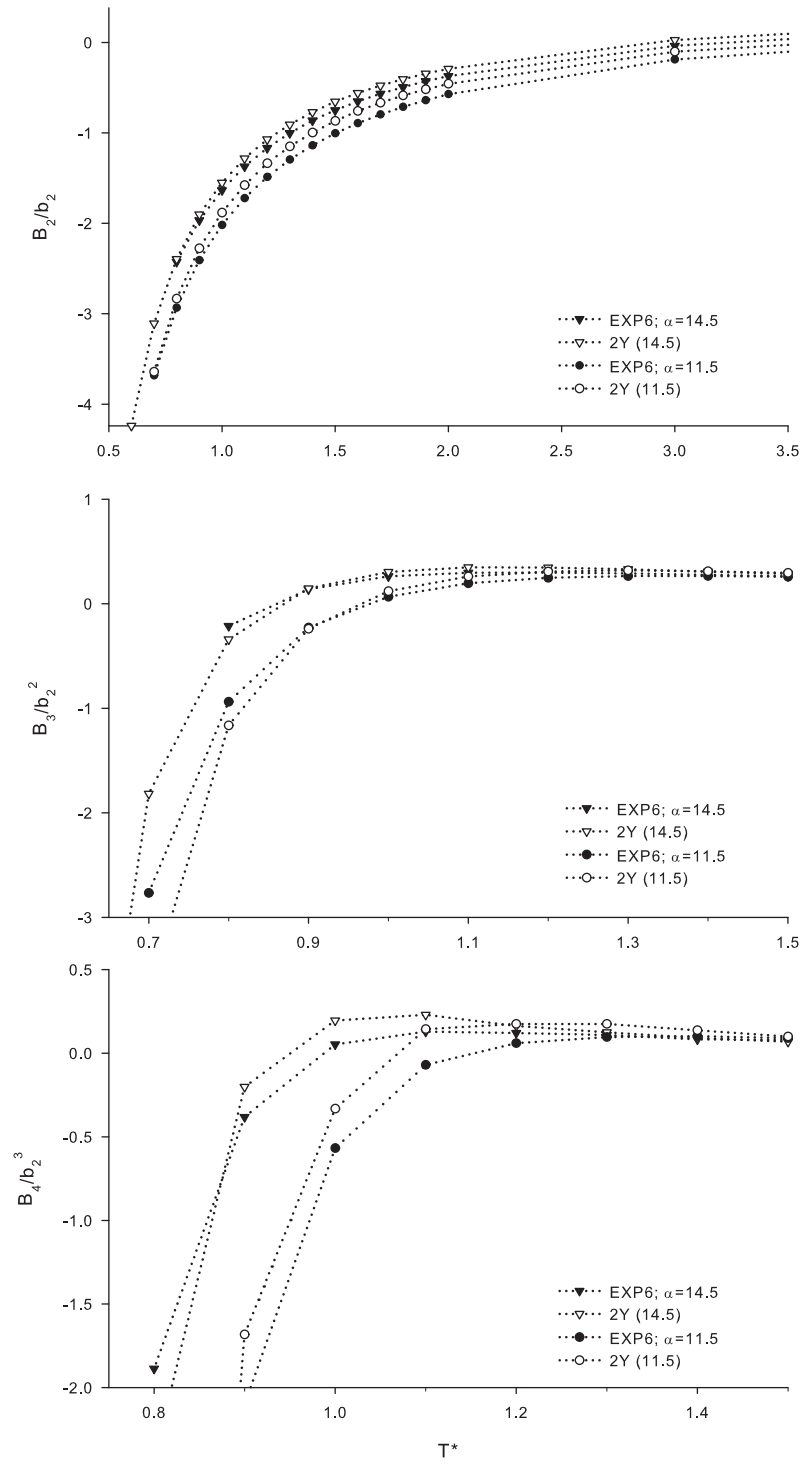

Figure 1. Comparison of the virial coefficients $B_{2}-B_{4}$ of the considered models. The dotted line has been drawn as a guide for eye. 
Table 2. Virial coefficients of the EXP6 potential with $\alpha=11.5 ; b_{2}=(2 / 3) \pi r_{\mathrm{m}}^{3}$.

\begin{tabular}{rccr}
\hline$T^{*}$ & $B_{2} / b_{2}$ & $B_{3} / b_{2}^{2}$ & $B_{4} / b_{2}^{3}$ \\
\hline 50.00 & $0.254 \pm 0.001$ & $0.049 \pm 0.001$ & $0.045 \pm 0.026$ \\
40.00 & $0.263 \pm 0.001$ & $0.057 \pm 0.001$ & $0.000 \pm 0.009$ \\
30.00 & $0.272 \pm 0.001$ & $0.066 \pm 0.001$ & $0.011 \pm 0.003$ \\
20.00 & $0.275 \pm 0.001$ & $0.080 \pm 0.001$ & $0.016 \pm 0.001$ \\
15.00 & $0.268 \pm 0.001$ & $0.090 \pm 0.001$ & $0.022 \pm 0.002$ \\
14.00 & $0.263 \pm 0.001$ & $0.092 \pm 0.001$ & $0.028 \pm 0.007$ \\
13.00 & $0.260 \pm 0.001$ & $0.094 \pm 0.001$ & $0.022 \pm 0.001$ \\
12.00 & $0.253 \pm 0.001$ & $0.097 \pm 0.001$ & $0.021 \pm 0.003$ \\
11.00 & $0.245 \pm 0.001$ & $0.100 \pm 0.001$ & $0.023 \pm 0.001$ \\
10.00 & $0.234 \pm 0.001$ & $0.103 \pm 0.001$ & $0.022 \pm 0.003$ \\
9.00 & $0.219 \pm 0.001$ & $0.106 \pm 0.001$ & $0.027 \pm 0.001$ \\
8.00 & $0.200 \pm 0.001$ & $0.111 \pm 0.001$ & $0.029 \pm 0.001$ \\
7.00 & $0.173 \pm 0.001$ & $0.115 \pm 0.001$ & $0.030 \pm 0.001$ \\
6.00 & $0.135 \pm 0.001$ & $0.120 \pm 0.001$ & $0.031 \pm 0.001$ \\
5.00 & $0.076 \pm 0.001$ & $0.127 \pm 0.001$ & $0.031 \pm 0.001$ \\
4.00 & $-0.018 \pm 0.001$ & $0.138 \pm 0.001$ & $0.031 \pm 0.001$ \\
3.00 & $-0.190 \pm 0.001$ & $0.158 \pm 0.001$ & $0.030 \pm 0.001$ \\
2.00 & $-0.573 \pm 0.001$ & $0.210 \pm 0.001$ & $0.046 \pm 0.002$ \\
1.90 & $-0.640 \pm 0.001$ & $0.219 \pm 0.001$ & $0.048 \pm 0.002$ \\
1.80 & $-0.714 \pm 0.001$ & $0.228 \pm 0.001$ & $0.057 \pm 0.002$ \\
1.70 & $-0.798 \pm 0.001$ & $0.239 \pm 0.001$ & $0.066 \pm 0.002$ \\
1.60 & $-0.895 \pm 0.001$ & $0.249 \pm 0.001$ & $0.076 \pm 0.002$ \\
1.50 & $-1.006 \pm 0.001$ & $0.258 \pm 0.001$ & $0.089 \pm 0.003$ \\
1.40 & $-1.139 \pm 0.001$ & $0.266 \pm 0.001$ & $0.101 \pm 0.004$ \\
1.30 & $-1.296 \pm 0.001$ & $0.264 \pm 0.001$ & $0.096 \pm 0.005$ \\
1.20 & $-1.489 \pm 0.001$ & $0.247 \pm 0.001$ & $0.059 \pm 0.006$ \\
1.10 & $-1.723 \pm 0.002$ & $0.196 \pm 0.001$ & $-0.071 \pm 0.009$ \\
1.00 & $-2.020 \pm 0.002$ & $0.066 \pm 0.002$ & $-0.569 \pm 0.014$ \\
0.90 & $-2.408 \pm 0.003$ & $-0.229 \pm 0.003$ & $-2.104 \pm 0.041$ \\
0.80 & $-2.934 \pm 0.003$ & $-0.939 \pm 0.004$ & $-7.230 \pm 0.230$ \\
0.70 & $-3.682 \pm 0.004$ & $-2.767 \pm 0.009$ & $-24.997 \pm 0.649$ \\
\hline & & &
\end{tabular}

Table 3. Virial coefficients of the EXP6 potential with $\alpha=14.5 ; b_{2}=(2 / 3) \pi r_{\mathrm{m}}^{3}$.

\begin{tabular}{rrcr}
\hline$T^{*}$ & $B_{2} / b_{2}$ & $B_{3} / b_{2}^{2}$ & $B_{4} / b_{2}^{3}$ \\
\hline 50.00 & $0.340 \pm 0.001$ & $0.083 \pm 0.001$ & $0.016 \pm 0.002$ \\
40.00 & $0.351 \pm 0.001$ & $0.091 \pm 0.001$ & $0.023 \pm 0.004$ \\
30.00 & $0.360 \pm 0.001$ & $0.103 \pm 0.001$ & $0.023 \pm 0.003$ \\
20.00 & $0.364 \pm 0.001$ & $0.116 \pm 0.001$ & $0.030 \pm 0.002$ \\
15.00 & $0.358 \pm 0.001$ & $0.126 \pm 0.001$ & $0.039 \pm 0.007$ \\
14.00 & $0.355 \pm 0.001$ & $0.129 \pm 0.001$ & $0.036 \pm 0.002$ \\
13.00 & $0.351 \pm 0.001$ & $0.131 \pm 0.001$ & $0.034 \pm 0.002$ \\
12.00 & $0.347 \pm 0.001$ & $0.135 \pm 0.001$ & $0.035 \pm 0.001$ \\
11.00 & $0.340 \pm 0.001$ & $0.135 \pm 0.001$ & $0.033 \pm 0.007$ \\
10.00 & $0.330 \pm 0.001$ & $0.138 \pm 0.001$ & $0.042 \pm 0.001$ \\
9.00 & $0.318 \pm 0.001$ & $0.142 \pm 0.001$ & $0.044 \pm 0.002$ \\
8.00 & $0.301 \pm 0.001$ & $0.144 \pm 0.001$ & $0.041 \pm 0.002$ \\
7.00 & $0.278 \pm 0.001$ & $0.147 \pm 0.001$ & $0.045 \pm 0.001$ \\
6.00 & $0.245 \pm 0.001$ & $0.150 \pm 0.001$ & $0.049 \pm 0.001$ \\
5.00 & $0.195 \pm 0.001$ & $0.154 \pm 0.001$ & $0.048 \pm 0.001$ \\
4.00 & $0.112 \pm 0.001$ & $0.160 \pm 0.001$ & $0.047 \pm 0.001$ \\
3.00 & $-0.039 \pm 0.001$ & $0.171 \pm 0.001$ & $0.043 \pm 0.001$ \\
2.00 & $-0.373 \pm 0.001$ & $0.209 \pm 0.001$ & $0.039 \pm 0.001$ \\
1.90 & $-0.429 \pm 0.001$ & $0.216 \pm 0.001$ & $0.041 \pm 0.001$ \\
1.80 & $-0.495 \pm 0.001$ & $0.225 \pm 0.001$ & $0.045 \pm 0.001$ \\
1.70 & $-0.568 \pm 0.001$ & $0.236 \pm 0.001$ & $0.051 \pm 0.002$ \\
1.60 & $-0.652 \pm 0.001$ & $0.249 \pm 0.001$ & $0.056 \pm 0.002$ \\
1.50 & $-0.751 \pm 0.001$ & $0.263 \pm 0.001$ & $0.078 \pm 0.006$ \\
1.40 & $-0.863 \pm 0.001$ & $0.276 \pm 0.001$ & $0.082 \pm 0.003$ \\
1.30 & $-1.003 \pm 0.001$ & $0.289 \pm 0.001$ & $0.108 \pm 0.004$ \\
1.20 & $-1.169 \pm 0.001$ & $0.297 \pm 0.001$ & $0.120 \pm 0.005$ \\
1.10 & $-1.373 \pm 0.002$ & $0.296 \pm 0.001$ & $0.128 \pm 0.007$ \\
1.00 & $-1.635 \pm 0.002$ & $0.262 \pm 0.002$ & $0.052 \pm 0.010$ \\
0.90 & $-1.969 \pm 0.002$ & $0.137 \pm 0.002$ & $-0.382 \pm 0.019$ \\
0.80 & $-2.427 \pm 0.002$ & $-0.213 \pm 0.002$ & $-1.888 \pm 0.160$ \\
\hline & & &
\end{tabular}


Table 4. Virial coefficients of the 2-Yukawa potential mimicking the EXP6 potential with $\alpha=$ $11.5 ; b_{2}=(2 / 3) \pi \sigma^{3}$.

\begin{tabular}{rrcc}
\hline$T^{*}$ & $B_{2} / b_{2}$ & $B_{3} / b_{2}^{2}$ & $B_{4} / b_{2}^{3}$ \\
\hline 50.00 & $0.268 \pm 0.001$ & $0.052 \pm 0.001$ & $0.007 \pm 0.001$ \\
40.00 & $0.278 \pm 0.001$ & $0.059 \pm 0.001$ & $0.010 \pm 0.001$ \\
30.00 & $0.290 \pm 0.001$ & $0.069 \pm 0.001$ & $0.013 \pm 0.001$ \\
20.00 & $0.297 \pm 0.001$ & $0.081 \pm 0.001$ & $0.017 \pm 0.001$ \\
15.00 & $0.293 \pm 0.001$ & $0.092 \pm 0.001$ & $0.019 \pm 0.003$ \\
14.00 & $0.291 \pm 0.001$ & $0.094 \pm 0.001$ & $0.022 \pm 0.001$ \\
13.00 & $0.287 \pm 0.001$ & $0.097 \pm 0.001$ & $0.023 \pm 0.001$ \\
12.00 & $0.282 \pm 0.001$ & $0.100 \pm 0.001$ & $0.015 \pm 0.013$ \\
11.00 & $0.276 \pm 0.001$ & $0.103 \pm 0.001$ & $0.024 \pm 0.001$ \\
10.00 & $0.268 \pm 0.001$ & $0.106 \pm 0.001$ & $0.025 \pm 0.001$ \\
9.00 & $0.257 \pm 0.001$ & $0.108 \pm 0.001$ & $0.028 \pm 0.001$ \\
8.00 & $0.241 \pm 0.001$ & $0.112 \pm 0.001$ & $0.030 \pm 0.001$ \\
7.00 & $0.218 \pm 0.001$ & $0.116 \pm 0.001$ & $0.031 \pm 0.001$ \\
6.00 & $0.186 \pm 0.001$ & $0.120 \pm 0.001$ & $0.032 \pm 0.001$ \\
5.00 & $0.134 \pm 0.001$ & $0.127 \pm 0.001$ & $0.032 \pm 0.001$ \\
4.00 & $0.051 \pm 0.001$ & $0.137 \pm 0.001$ & $0.032 \pm 0.001$ \\
3.00 & $-0.104 \pm 0.001$ & $0.159 \pm 0.001$ & $0.028 \pm 0.001$ \\
2.00 & $-0.458 \pm 0.001$ & $0.223 \pm 0.001$ & $0.038 \pm 0.001$ \\
1.90 & $-0.520 \pm 0.001$ & $0.234 \pm 0.001$ & $0.042 \pm 0.001$ \\
1.80 & $-0.588 \pm 0.001$ & $0.249 \pm 0.001$ & $0.051 \pm 0.002$ \\
1.70 & $-0.669 \pm 0.001$ & $0.262 \pm 0.001$ & $0.068 \pm 0.005$ \\
1.60 & $-0.760 \pm 0.001$ & $0.278 \pm 0.001$ & $0.087 \pm 0.003$ \\
1.50 & $-0.870 \pm 0.001$ & $0.295 \pm 0.001$ & $0.099 \pm 0.003$ \\
1.40 & $-0.998 \pm 0.001$ & $0.310 \pm 0.001$ & $0.136 \pm 0.004$ \\
1.30 & $-1.151 \pm 0.001$ & $0.319 \pm 0.001$ & $0.174 \pm 0.006$ \\
1.20 & $-1.338 \pm 0.002$ & $0.307 \pm 0.001$ & $0.174 \pm 0.008$ \\
1.10 & $-1.580 \pm 0.002$ & $0.260 \pm 0.002$ & $0.143 \pm 0.012$ \\
1.00 & $-1.883 \pm 0.002$ & $0.119 \pm 0.002$ & $-0.333 \pm 0.021$ \\
0.90 & $-2.278 \pm 0.002$ & $-0.240 \pm 0.003$ & $-1.683 \pm 0.308$ \\
0.80 & $-2.834 \pm 0.003$ & $-1.165 \pm 0.004$ & $-8.430 \pm 0.150$ \\
\hline & & & \\
\hline
\end{tabular}

Table 5. Virial coefficients of the 2-Yukawa potential mimicking the EXP6 potential with $\alpha=$ $14.5 ; b_{2}=(2 / 3) \pi \sigma^{3}$.

\begin{tabular}{rrrr}
\hline$T^{*}$ & $B_{2} / b_{2}$ & $B_{3} / b_{2}^{2}$ & $B_{4} / b_{2}^{3}$ \\
\hline 50.00 & $0.355 \pm 0.001$ & $0.087 \pm 0.001$ & $0.015 \pm 0.002$ \\
40.00 & $0.363 \pm 0.001$ & $0.095 \pm 0.001$ & $0.027 \pm 0.006$ \\
30.00 & $0.376 \pm 0.001$ & $0.106 \pm 0.001$ & $0.021 \pm 0.002$ \\
20.00 & $0.383 \pm 0.001$ & $0.120 \pm 0.001$ & $0.031 \pm 0.001$ \\
15.00 & $0.380 \pm 0.001$ & $0.130 \pm 0.001$ & $0.028 \pm 0.006$ \\
14.00 & $0.378 \pm 0.001$ & $0.131 \pm 0.001$ & $0.036 \pm 0.002$ \\
13.00 & $0.374 \pm 0.001$ & $0.134 \pm 0.001$ & $0.035 \pm 0.004$ \\
12.00 & $0.371 \pm 0.001$ & $0.135 \pm 0.001$ & $0.039 \pm 0.001$ \\
11.00 & $0.365 \pm 0.001$ & $0.137 \pm 0.001$ & $0.046 \pm 0.005$ \\
10.00 & $0.359 \pm 0.001$ & $0.140 \pm 0.001$ & $0.042 \pm 0.001$ \\
9.00 & $0.348 \pm 0.001$ & $0.143 \pm 0.001$ & $0.042 \pm 0.001$ \\
8.00 & $0.334 \pm 0.001$ & $0.145 \pm 0.001$ & $0.045 \pm 0.001$ \\
7.00 & $0.314 \pm 0.001$ & $0.147 \pm 0.001$ & $0.046 \pm 0.001$ \\
6.00 & $0.284 \pm 0.001$ & $0.150 \pm 0.001$ & $0.049 \pm 0.001$ \\
5.00 & $0.239 \pm 0.001$ & $0.153 \pm 0.001$ & $0.049 \pm 0.001$ \\
4.00 & $0.163 \pm 0.001$ & $0.158 \pm 0.001$ & $0.047 \pm 0.001$ \\
3.00 & $0.026 \pm 0.001$ & $0.168 \pm 0.001$ & $0.041 \pm 0.001$ \\
2.00 & $-0.292 \pm 0.001$ & $0.214 \pm 0.001$ & $0.033 \pm 0.001$ \\
1.90 & $-0.345 \pm 0.001$ & $0.225 \pm 0.001$ & $0.031 \pm 0.001$ \\
1.80 & $-0.407 \pm 0.001$ & $0.236 \pm 0.001$ & $0.038 \pm 0.002$ \\
1.70 & $-0.478 \pm 0.001$ & $0.250 \pm 0.001$ & $0.043 \pm 0.002$ \\
1.60 & $-0.560 \pm 0.001$ & $0.268 \pm 0.001$ & $0.050 \pm 0.002$ \\
1.50 & $-0.655 \pm 0.001$ & $0.286 \pm 0.001$ & $0.068 \pm 0.002$ \\
1.40 & $-0.771 \pm 0.001$ & $0.306 \pm 0.001$ & $0.091 \pm 0.004$ \\
1.30 & $-0.909 \pm 0.001$ & $0.329 \pm 0.001$ & $0.124 \pm 0.005$ \\
1.20 & $-1.074 \pm 0.001$ & $0.345 \pm 0.001$ & $0.162 \pm 0.006$ \\
1.10 & $-1.283 \pm 0.001$ & $0.348 \pm 0.001$ & $0.229 \pm 0.010$ \\
1.00 & $-1.553 \pm 0.001$ & $0.304 \pm 0.002$ & $0.195 \pm 0.015$ \\
0.90 & $-1.904 \pm 0.002$ & $0.146 \pm 0.002$ & $-0.202 \pm 0.023$ \\
0.80 & $-2.397 \pm 0.002$ & $-0.342 \pm 0.003$ & $-2.433 \pm 0.056$ \\
0.70 & $-3.108 \pm 0.003$ & $-1.818 \pm 0.007$ & $-14.300 \pm 0.330$ \\
0.60 & $-4.239 \pm 0.004$ & $-6.815 \pm 0.025$ & $-86.259 \pm 2.362$ \\
\hline & & & \\
\hline
\end{tabular}


Numerical values of the virial coefficients of all four fluids considered are given in tables 2 through 5 and are also compared in figure 1. Examination of the tables/figure shows that the coefficients of the EXP6 and $2 \mathrm{Y}$ fluids are very similar. This is a consequence of the fact that the repulsive parts of the EXP6 and $2 \mathrm{Y}$ models have been used to fit the corresponding 2nd virial coefficients [2]. In other words, the similarity of all the virial coefficients means that the long-range part of the models does not affect them to any important extent.

Table 6. Vapor-liquid equilibrium data of the $2 \mathrm{Y}$ fluid mimicking the EXP6 potential with $\alpha=11.5$.

\begin{tabular}{ccc}
\hline$T^{*}$ & $\rho_{v}^{*}$ & $\rho_{l}^{*}$ \\
\hline 0.700 & $0.0073 \pm 0.0031$ & $1.1798 \pm 0.0130$ \\
0.800 & $0.0194 \pm 0.0060$ & $1.1097 \pm 0.0162$ \\
0.900 & $0.0438 \pm 0.0086$ & $1.0331 \pm 0.0177$ \\
1.000 & $0.0886 \pm 0.0159$ & $0.9327 \pm 0.0294$ \\
1.030 & $0.1107 \pm 0.0183$ & $0.9012 \pm 0.0352$ \\
1.050 & $0.1333 \pm 0.0253$ & $0.8748 \pm 0.0387$ \\
1.070 & $0.1567 \pm 0.0288$ & $0.8540 \pm 0.0353$ \\
1.100 & $0.1930 \pm 0.0439$ & $0.7805 \pm 0.0610$ \\
1.120 & $0.2324 \pm 0.0282$ & $0.7203 \pm 0.0577$ \\
1.140 & $0.2732 \pm 0.0502$ & $0.6241 \pm 0.0717$ \\
\hline
\end{tabular}

Table 7. Vapor-liquid equilibrium data of the $2 \mathrm{Y}$ fluid mimicking the EXP6 potential with $\alpha=14.5$.

\begin{tabular}{ccc}
\hline$T^{*}$ & $\rho_{v}^{*}$ & $\rho_{l}^{*}$ \\
\hline 0.600 & $0.0054 \pm 0.0028$ & $1.1571 \pm 0.0113$ \\
0.700 & $0.0172 \pm 0.0049$ & $1.0863 \pm 0.0152$ \\
0.800 & $0.0454 \pm 0.0105$ & $0.9983 \pm 0.0193$ \\
0.850 & $0.0688 \pm 0.0132$ & $0.9437 \pm 0.0219$ \\
0.900 & $0.1055 \pm 0.0167$ & $0.8799 \pm 0.0283$ \\
0.930 & $0.1394 \pm 0.0251$ & $0.8287 \pm 0.0459$ \\
0.950 & $0.1533 \pm 0.0256$ & $0.7852 \pm 0.0603$ \\
0.970 & $0.1948 \pm 0.0257$ & $0.7512 \pm 0.0462$ \\
0.975 & $0.2072 \pm 0.0319$ & $0.7287 \pm 0.0576$ \\
0.980 & $0.2157 \pm 0.0341$ & $0.7078 \pm 0.0584$ \\
0.985 & $0.2211 \pm 0.0348$ & $0.6920 \pm 0.0604$ \\
0.990 & $0.2320 \pm 0.0238$ & $0.6677 \pm 0.0527$ \\
0.995 & $0.2474 \pm 0.0548$ & $0.6012 \pm 0.0720$ \\
\hline
\end{tabular}

Table 8. Critical properties of the $2 \mathrm{Y}$ fluids determined from the vapor-liquid coexistence data. Numbers in parentheses give results of the perturbed virial expansion (first row) and the virial expansion (second row) of the second order.

\begin{tabular}{ccr}
\hline Model & $\rho^{*}$ & \multicolumn{1}{c}{$T^{*}$} \\
\hline 2Y-11.5 & 0.452 & 1.171 \\
& & $(1.18)$ \\
& & $(1.43)$ \\
$2 \mathrm{Y}-14.5$ & 0.295 & 1.00 \\
& & $(1.05)$ \\
& & $(1.24)$ \\
\hline
\end{tabular}

The VLE results for the equilibrium densities of the $2 \mathrm{Y}$ fluids are given in tables 6 and 7 . With these data, the critical point was determined using the rectilinear rule and the common analytic parametrization expression

$$
\rho_{\mathrm{l}}-\rho_{\mathrm{v}}=B_{0}|t|^{\beta}+B_{1}|t|^{\beta+\Delta_{1}},
$$

and

$$
\frac{\rho_{\mathrm{l}}+\rho_{\mathrm{v}}}{2}=\rho_{\mathrm{c}}+C_{1}|t|^{\psi}+C_{2}|t|+C_{3}|t|^{\psi+\Delta_{1}}
$$

where $t=1-T / T_{\mathrm{C}}$ and $B_{i}, C_{i}, \beta$ and $\psi$ are parameters to be fitted to the simulation data; $\Delta_{1}=0.5$ for the vapor-liquid equilibria [12]. The results are given in table 8 . In this table we also 
give an estimate of the critical temperature obtained using the common virial expansion and the perturbed virial expansion of the 2nd order [13, 14]; in the perturbed expansion we used the fluid of hard spheres of diameter $\sigma$ as the reference. As we see, the perturbed method provides a very good estimate which further confirms its superiority over the common virial expansion.

\section{Conclusions}

In this paper we have presented results for the first four virial coefficients of the EXP6 fluid and the associated 2-Yukawa fluids, and vapor-liquid equilibria in the latter models. These data are necessary for a subsequent development of a theory for the 2-Yukawa fluids which further provides an alternative to hard core (van der Waals type) equations of state. In addition to the determination of the critical point from the vapor-liquid coexistence data, we have used the computed virial coefficients to determine the critical temperature from the virial expansions. The obtained results further confirm the recently reported applicability and accuracy of the perturbed expansion.

\section{Acknowledgement}

This work was supported by the Grant Agency of the Academy of Sciences of the Czech Republic (Grant No. IAA400720710), by the Grant Agency of the J.E. Purkinje University (Grant No. 5322315-0010-01), and the Czech-Ukrainian Bilateral Cooperative Program.

\section{References}

1. Ross M., Ree F.H., J. Chem. Phys., 1980, 73, 6146-6152; doi 10.1063/1.440106

2. Krejčí J., Nezbeda I., Melnyk R., Trokhymchuk A., J. Chem. Phys., 2010, 113, 094503:1-8; doi: $10.1063 / 1.3478220$.

3. Nezbeda I., Melnyk R., Trokhymchuk A., J. Supercrit. Fluids, 2010, 55, 448-454; doi: $10.1016 /$ j.supflu.2010.10.041

4. Rosenfeld Y., J. Chem. Phys., 1993, 98, 8126-8148; doi 10.1063/1.464569

5. Shukla K.P., J. Chem. Phys., 2000, 112, 10358-10367; doi 10.1063/1.481673

6. Duda Y., Romero-Martínez A., Orea P., J. Chem. Phys., 2007, 126, 224510:1-4; doi: $10.1063 / 1.2743623$.

7. Naresh D.J., Singh J.K., Fluid Phase Equil., 2009, 285, 36-43; doi 10.1016/j.fluid.2009.06.019

8. Kalyuzhnyi Yu., Cummings P.T., Mol. Phys., 1996, 87, 1459-1462; doi 10.1080/00268979600100981

9. Sun J.X., Mol. Phys., 2007, 105, 3139-3144; doi 10.1080/00268970701769938.

10. Singh J.K., Kofke D.A., Phys. Rev. Lett., 2004, 92, 220601:1-4; doi 10.1103/PhysRevLett.92.220601.

11. Vortler H.L., Nezbeda I., Lisal M., Mol. Phys., 1997, 92, 813-824; doi 10.1080/00268979709482153.

12. Vega L., de Miguel E., Rull L.F., Jackson G., McLure I.A., J. Chem. Phys., 1992, 96, 2296-2305; doi: $10.1063 / 1.462080$.

13. Nezbeda I., Smith W.R., Fluid Phase Equil., 2004, 216, 183-186; doi 10.1016/j.fluid.2003.11.006

14. Krejčí J., Nezbeda, Fluid Phase Equil., submitted. 


\title{
Віріальні коефіцієнти і фазова рівновага пара-рідина у EXP6
} та 2-Юкава плинах

\author{
Я. Крейч奋, І. Незбеда $2^{112}$, Р. Мельник ${ }^{3}$, А. Трохимчук ${ }^{3}$
}

1 Факультет науки, Університет Я.Е. Пуркінйє, 40096 Усті над Лабем, Чеська Республіка

2 Інститут фундаментальних основ хімічних процесів, Академія наук, 16502 Прага 6,

Чеська Республіка

3 Інститут фізики конденсованих систем НАН України, 79011 Львів, вул. Свєнціцького, 1

Віріальні коефіцієнти від $B_{2}$ до $B_{4}$ і фазова рівновага пара-рідина у EXP6 та 2-Юкава (2Y) плинах розраховані, відповідно, з допомогою чисельного інтегрування та на основі комп'ютерного експерименту з використанням ансамблю Гібса. Вибрані 2Ү модельні системи нещодавно були запропоновані як базисні для ЕXР6 плинів, що розглядаються.

Ключові слова: ЕХР6 плин, 2-Юкава плин, віріальні коефіцієнти, фазова рівновага пара-рідина, критична точка 